\title{
Vortex tubes in the wave bottom boundary layer
}

\author{
M. Henriquez ${ }^{1}$, A.J.H.M. Reniers ${ }^{2}$, B.G. Ruessink ${ }^{3}$ and M.J.F. Stive ${ }^{4}$ \\ ${ }^{1}$ Hydraulic Engineering, Delft University of Technology, P.O. Box 5048, 2600 GA, Delft, The Netherlands, m.henriquez@tudelft.nl \\ ${ }^{2}$ Rosenstiel School of Marine and Atmospheric Science, University of Miami, 4600 Rickenbacker Causeway, Miami, FL 33149, \\ USA \\ ${ }^{3}$ Physical Geography, Utrecht University, Heidelberglaan 2, 3584 CS, Utrecht, The Netherlands \\ ${ }^{4}$ Hydraulic Engineering, Delft University of Technology, P.O. Box 5048, 2600 GA, Delft, The Netherlands
}

\begin{abstract}
The cause of sediment suspension events during flow reversal under waves in the nearshore is not well understood. Vortex tubes and horizontal pressure gradients have been suggested to be the cause of the suspension events. A medium sized wave flume experiment has been conducted to give insight in the hydrodynamics of the wave bottom boundary layer over a fixed single-barred profile. Flow measurements were made with PIV and the swirling strength of the velocity fields were analyzed. Around flow reversal vortex tubes were identified. The vortex tubes had similar size and swirling strength as vortices generated by vortex shedding over a rippled bed. Therefore, vortex tubes under waves in the nearshore could explain the sediment suspension events around flow reversal.
\end{abstract}

\section{INTRODUCTION}

The wave bottom boundary layer exists due to friction between the orbital fluid motion and the bottom. The relatively thin layer plays an important role in the sediment transport by waves in the nearshore. In the layer, sediment is mobilized and transported in various ways, for instance, as sheet flow. There are also observations of sudden suspension events around the reversal of the flow as described in Madsen [1974], "just prior to the passage of the crest of a near-breaking wave the bottom seemed to explode". Often pressure gradients are suggested to cause bed failure around flow reversal (see for example Madsen, [1974]; Drake and Calantoni, [2001]; Hoefel and Elgar, [2003]; Zala Flores and Sleath, [1998]). Although pressure gradients provide additional forces on sediment particles to aid mobility it does not explain the suspension event (it would rather explain liquefaction of the bed). Foster et al. [1994] suggested that the coherent structures, such as vortex tubes [Carstensen et al. 2010], are generated in the wave bottom boundary layer under surface waves in the nearshore and could be responsible for sediment suspension events. Vortex tubes are instabilities generated at an inflectionalpoint of an oscillatory boundary layer. They were observed in the bottom boundary layer of oscillating flow tunnels at flow reversal (see for example Akhavan et al. [1991] and Carstensen et al. [2010]). Cartensen et al. [2010] conducted experiments in an oscillating flow tunnel with a smooth fixed bed and concluded that the impact of the vortex tubes on the bottom shear stress is insignificant. Still, vortex tubes provide a mechanism for the advection of sediment during flow reversal. If, indeed, vortex tubes generate suspension events around flow reversal, the contribution of horizontal pressure gradients to the force balance on sediment particles in the nearshore remains unclear.

Recently, vortex tubes were detected in a wave flume experiment with a fixed bed. The relation between vortex tubes and suspension events is researched by measuring the swirling strength and size of the vortex tubes.

\section{EXPERIMENT}

The flume has a length of $40 \mathrm{~m}$, a width of $0.8 \mathrm{~m}$ and a water depth of $0.5 \mathrm{~m}$. In the flume a rigid single bar profile was build. The top of the bar is approximately $0.15 \mathrm{~m}$ below the mean water level. Granular sediment with a grain size of $0.54 \mathrm{~mm}$ was glued to the surface to provide bottom roughness. The hydrodynamic model scale was 1:10 which corresponds to a medium sized wave flume experiment.

The vertical and horizontal flow velocities within the wave bottom boundary layer were measured with Particle Image Velocimetry (PIV) at several locations along the flume [Henriquez et al. 2010]. For this study, we limit ourselves to one location just before the bar crest (taken that the wave maker is the origin) and one wave condition. At this location, the water depth was $0.154 \mathrm{~m}$, the root-mean-square wave height was $0.087 \mathrm{~m}$ and the wave period of the regular waves was $1.8 \mathrm{~s}$. The wave field consisted of 120 waves. PIV measurements were conducted at a rate of $15 \mathrm{~Hz}$ resulting in 27 different phases in one wave cycle.

The laser sheet for PIV was inserted into the water from the water surface using a streamlined window. The camera was placed outside of the flume (flume wall is transparent). The camera had a field of view of approximately $10 \times 10 \mathrm{~mm}^{2}$. The camera images were processed resulting in a velocity vector for every $0.37 \times 0.37$ $\mathrm{mm}^{2}$. The flow velocity vector is decomposed into a horizontal component $u$, positive in wave direction, and a vertical component $w$, positive upwards.

Figure 1 shows the phase-average horizontal velocity $\hat{u}$ at the upper boundary of the velocity vector field which is approximately in the free-stream (outside the wave bottom boundary layer). The shape of the horizontal velocity is skewed and asymmetric indicating that the waves were shoaling. 


\section{SWIRLING STRENGTH}

Vortices in a shear flow can be identified and quantified with the eigenvalues of the velocity gradient tensor [Adrian et al. 2000]. PIV measurements are usually two-dimensional, e.g. in the $x z$-plane, resulting in the velocity gradient tensor:

$$
\mathbf{D}=\left(\begin{array}{ll}
\frac{\partial u}{\partial x} & \frac{\partial u}{\partial z} \\
\frac{\partial w}{\partial x} & \frac{\partial w}{\partial z}
\end{array}\right)
$$

The characteristic equation of $\mathbf{D}$ is then

$$
\lambda^{2}+P \lambda+Q
$$

where $P=-\operatorname{tr}(\mathbf{D})$ and $Q=\operatorname{det}(\mathbf{D})$. When written out, $P$ becomes

$$
P=-\left(\frac{\partial u}{\partial x}+\frac{\partial w}{\partial z}\right)
$$

and $Q$ becomes

$$
Q=\frac{\partial u}{\partial x} \frac{\partial w}{\partial z}-\frac{\partial u}{\partial z} \frac{\partial w}{\partial x}
$$

The solutions of the characteristic equation are the eigenvalues of the matrix $\mathbf{D}$. Whether the eigenvalues are complex or real can be determined with the discriminant $\Delta=P^{2}-4 Q$ and when written out becomes

$$
\Delta=\frac{\partial u^{2}}{\partial x}+\frac{\partial w^{2}}{\partial z}-2 \frac{\partial u}{\partial x} \frac{\partial w}{\partial z}+4 \frac{\partial u}{\partial z} \frac{\partial w}{\partial x}
$$

Now, complex eigenvalues of the velocity gradient tensor indicate the presence of a vortex. Thus, a negative discriminant ( $\Delta<0$ ) indicates the presence of a vortex. The spiraling motion of a vortex can be quantified by the swirling strength $\lambda_{c i}$ which is the imaginary part of the complex eigenvalue pair [Zhou et al. 1999]. The square of the swirling strength is proportional to the

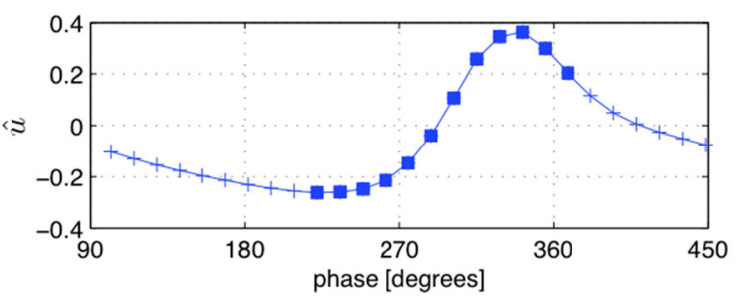

Figure 1. Phase-averaged horizontal velocity at the upper boundary of the velocity vector field. Horizontal axis is phase in degrees and vertical axis is velocity in $\mathrm{m} / \mathrm{s}$. The square markers refer to the phases that are shown in Figure 2. discriminant $\left(4 \lambda_{c i}^{2}=-\Delta\right)$. The value of $\lambda_{c i}^{-1}$ represents the period required to spiral around the origin of the vortex [Adrian et al. 2000].

\section{RESULTS}

The swirling strength was calculated for all vector fields. Areas with a swirling strength of $\geq 5 \mathrm{~s}^{-11}$ were identified as vortices. Statistical properties of vortices such as size, equivalent diameter, average swirling strength and location of centroid were determined for every wave phase.

Generally, the number of vortices decreases exponentially with increasing vortex diameter and, on average, swirling strength of a vortex was between 10 and $20 \mathrm{~s}^{-1}$. Quantities such as average swirling strength, number of vorticies and maximum vortex size were larger during positive free-stream velocities than during negative free-stream velocities.

Remarkable was the generation of relatively large vortices with diameters of 3 to $4 \mathrm{~mm}$ and an average swirling strength between 15 and $20 \mathrm{~s}^{-1}$ at free-stream flow reversal from negative to positive. These vortices were generated close to the bed and originate from one specific location. The consistent temporal and spatial behavior of vortices at one specific location results in the presence of a vortex in the phase-averaged flow field, i.e. it will not be averaged out. Figure 2 shows the appearance of the vortex at phase $275^{\circ}$ just prior to flow reversal (which is at phase $288^{\circ}$ ). After flow reversal, at phase $302^{\circ}$, the swirling strength of the vortex decreases and the shape is less pronounced. At phases $315^{\circ}, 328^{\circ}$ and $342^{\circ}$ relatively large patches of high swirling strength are still visible and it is suspected that these originated at locations outside the measuring domain and were transported by the flow.

The fact that the origin coincided with an inflectional-point of a shear layer suggests that these vortices were vortex tubes. Irregularities in the bottom profile of the fixed bed could explain the persistent location of the origin. To put the vortex tube in perspective we use the experimental results of Nichols and Foster [2007] who measured the flow field of surface waves above an irregularly rippled movable bed (wavelength $\approx 0.1 \mathrm{~m}$ and height $\approx 0.01 \mathrm{~m}$ and found vortices, generated by vortex shedding, with swirling strengths of $\approx 10 \mathrm{~s}^{-1}$ and diameters of $\approx 4 \mathrm{~mm}$. It was observed that a sediment plume was entrained into such a vortex. The vortices identified by Nichols and Foster [2007] are of similar size and swirling strength as the vortices of this study. This makes it likely that the vortex tubes from the experiment described herein were able to suspend sediment.

\section{CONCLUSIONS}

Coherent structures, i.e. vortices, were detected in the wave bottom boundary layer of a medium sized wave flume using swirling strength. The temporal and spatial behavior of some vortices suggested that these were vortex tubes generated by an inflectional-point of a shear layer during flow reversal. Comparison of the vortex tubes with vortices generated by vortex shedding over ripples showed similar swirling strength and size indicating the ability of vortex tubes to cause sediment suspension events around flow reversal. The contribution of horizontal pressure gradients to sediment transport in the nearshore remains unclear. 

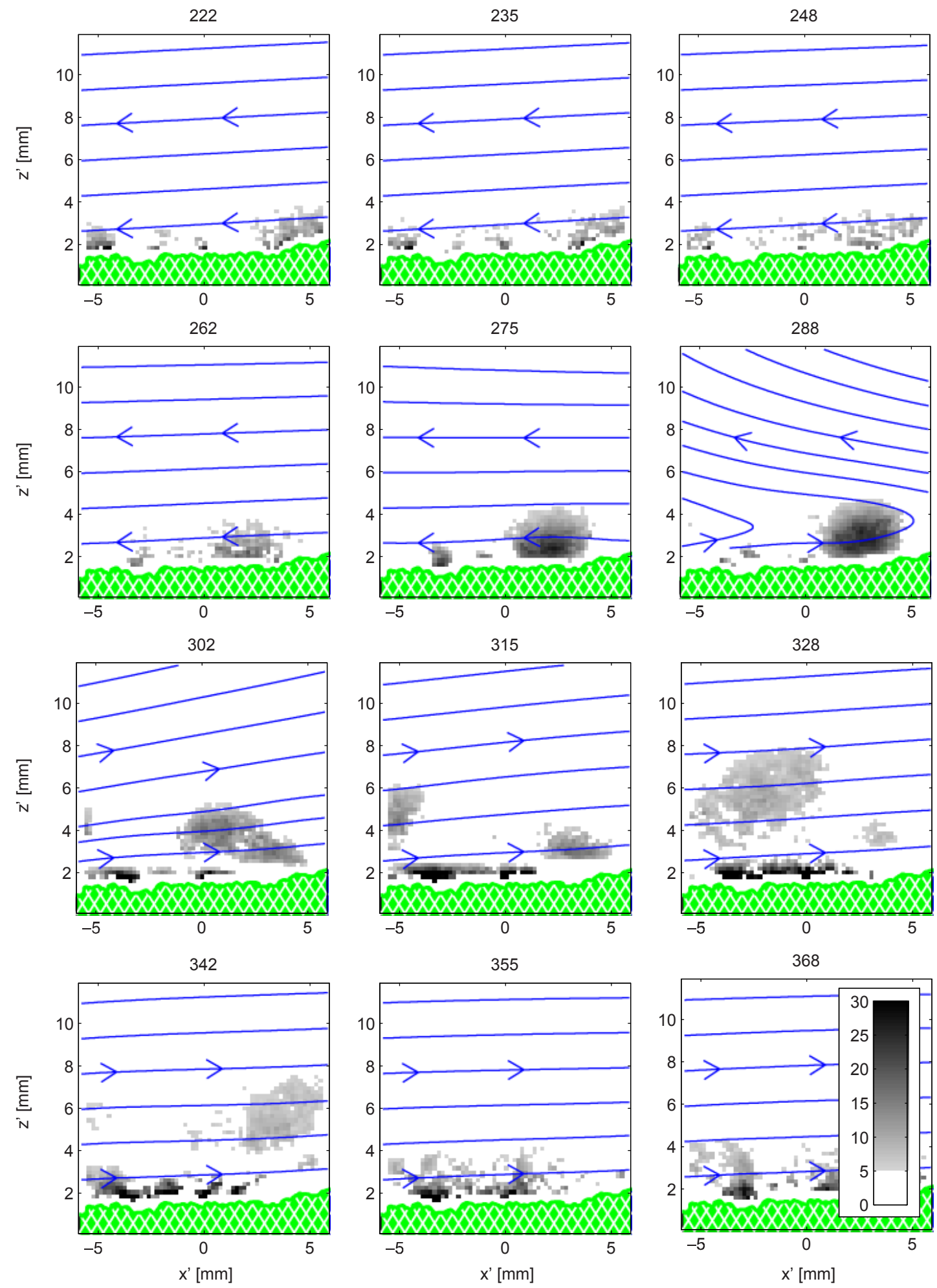

Figure 2. Swirling strength of the phase-average velocity during flow reversal. Color bar indicates swirling strength. Solid blue lines represent streamlines of the flow and green hatched area represents the bottom. The horizontal axis is horizontal distance and the vertical axis is vertical distance with the origin at the center bottom of the PIV vector field. The numbers above subfigures are the wave phases in degrees and correspond with the square markers in Figure 1. 
Further research is needed to investigate the existence of vortex tubes at real scales in the nearshore and their role in net sediment transport.

\section{ACKNOWLEDGEMENT}

This project is funded by the Technology Foundation STW, applied science division of NWO, in The Netherlands, under project number DCB.07908. The authors are presently supported by the ERC Advanced Grant 291206 - NEMO.

\section{REFERENCES}

Adrian, R. J., K. T. Christensen, and Z.-C. Liu. (2000). Analysis and interpretation of instantaneous turbulent velocity fields. Experiments in Fluids 29 (3) (september): 275-290. doi: $10.1007 / \mathrm{s} 003489900087$.

Akhavan, R., R. D. Kamm, and A. H. Shapiro (2006). An investigation of transition to turbulence in bounded oscillatory Stokes flows Part 1. Experiments. Journal of Fluid Mechanics 225 (-1) (april): 395. doi:10.1017/S0022112091002100.

Carstensen, S., B. M. Sumer, and J. Fredsøe. (2010). Coherent structures in wave boundary layers. Part 1. Oscillatory motion. Journal of Fluid Mechanics 646 (March): 169. doi:10.1017/S0022112009992825.

Drake, T. G., and J. Calantoni. (2001). Discrete particle model for sheet flow sediment transport in the nearshore. Journal of Geophysical Research 106 (C9): 868.
Foster, D. L., R. Holman, and R. A. Beach (1994). Sediment suspension events and shear instabilities in the bottom boundary layer. In Proceedings Coastal Dynamics 94, 712-726.

Henriquez, M., A. J. H. M. Reniers, B. G. Ruessink, and M. J. F. Stive (2010). Wave boundary layer hydrodynamics during onshore bar migration. In Proceedings of the 32nd International Conference on Coastal Engineering, Vol. 1, Shanghai, China.

Hoefel, F., and S. Elgar (2003). Wave-Induced Sediment Transport and Sandbar Migration. Science 299 (5614) (maart 21): 1885 -1887. doi:10.1126/science. 1081448.

Madsen, O. S. (1974). Stability of a sand bed under breaking waves. In Proceedings of the 14th International Conference on Coastal Engineering, 14:776.

Nichols, C. S., and D. L. Foster (2007). Full-scale observations of wave-induced vortex generation over a rippled bed. Journal of Geophysical Research 112 (oktober 13): 17 PP. doi:200710.1029/2006JC003841.

Zala Flores, N., and J. F. A. Sleath (1998). Mobile layer in oscillatory sheet flow. Journal of Geophysical Research 103: 12783-12793. doi:10.1029/98JC00691.

Zhou, J., R. J. Adrian, S. Balachandar, and T. M. Kendall (1999). Mechanisms for Generating Coherent Packets of Hairpin Vortices in Channel Flow. Journal of Fluid Mechanics 387: 353-396. 